\title{
À propos des lectures des questions en comment
}

\author{
Damien Fleury ${ }^{1}$, et Lucia M. Tovena ${ }^{1}$ \\ ${ }^{1}$ Université Paris Diderot - Paris 7, 5 rue Thomas Mann, 75205 Paris cedex 13, France
}

Résumé. Cette contribution porte sur les interprétations des interrogatives introduites par comment, qui peuvent interroger sur la manière mais aussi sur la cause, ou donner lieu à des questions parfois qualifiées de rhétoriques. Nous observons des affinités entre les prédicats qui enchâssent la préjacente dans une lecture de cause et les prédicats à montée de la négation et les prédicats factifs. Nous montrons comment plusieurs facteurs du sémantisme de ces prédicats orientent l'interprétation de la question dans une palette d'interprétations qui ne constitue pas pour autant un continuum. Il y a rupture entre les interprétations de manière, de cause et la lecture rhétorique. L'appellation 'rhétorique' se trouve enrichie de contenu du fait que la question du statut de la préjacente est abordée à l'intérieur d'une vue du contexte en tant que connaissances/croyances des agents épistémiques présents dans la situation. Nous montrons que l'interprétation des questions en comment de cause, qu'elles soient 'rhétoriques' ou non, se fonde sur l'établissement d'une polarité de jugements, inhérente à ce type de question, entre un jugement sur la préjacente et un jugement sur la proposition modale. Les informations du contexte permettent alors l'assignation des valeurs de cette polarité et in fine l'interprétation de la question en comment.

\begin{abstract}
About the readings of questions with comment ('how'). This paper is about the readings available for wh- questions containing the whword comment (how), which can be manner questions, but also be about causes of state of affairs, or have seeming rhetorical interpretations. We underscore some affinities between the predicates that embed the prejacent in a causal reading, and neg-raising or factive predicates. It is shown how several components of the meaning of these predicates can drive the interpretation of the question in a range of readings that do not form a continuum. There is a break between the readings of manner, of cause and the rhetorical reading. The issue of the status of the prejacent is tackled within a treatment of the context as composed of knowledge and beliefs of the epistemic agents present in the situation, which yields a more informative content for the term of 'rhetorical' as a result. It is shown that the causl interpretation of the questions with comment, be they rhetorical or ordinary, is grounded in setting up a polarity between two judgements, one about the prejacent and one about the modal proposition, which is inherent to this type of question. This setting makes contextual information sufficient for assigning the values of the polarity and finally for interpreting the question with comment.
\end{abstract}




\section{Introduction}

Les phrases interrogatives avec ce que l'on peut appeler le mot qu-comment sont interprétées comme des questions partielles sur la manière du déroulement d'une action ou le moyen/instrument utilisé pour l'accomplir, voir (1a,b). De plus, elles admettent d'autres interprétations, classifiées de différentes manières dans la production scientifique. De Cornulier (1974), Korzen (1990) et Fleck (2008) parlent de lecture causale, lorsque la réponse est congruente avec une interprétation de comment qui prend portée sur toute la phrase, voir $(1 \mathrm{a}, \mathrm{c})$. Un phénomène analogue a été observé pour d'autres langues, voir Collins (1991), Tsai (2008), Hsiao (2017) et d'autres, et on y fait parfois référence par le terme d'alternance pourquoi/comment.

(1) a. Comment Jean a-t-il survécu ?

b. En buvant du lait

(moyen)

c. C'est qu'il est résistant

(cause)

Desmets et Gautier (2009) parlent d'interprétation rhétorique lorsqu'il n'y aurait pas de réponse congruente avec une interprétation de comment de manière, voir la paire sous $(2 a, b)$, mais que la proposition enchâssée est comprise comme étant assertée, voir la paraphrase en (2c). Cependant, (2a) admet une lecture causale où un opérateur qu- lie une variable de proposition, ce qui est illustré par la congruence de (2a) avec la réponse en $(2 \mathrm{~d})$.

(2) a. Comment n'avez-vous pas reçu ma lettre ?

b. \# En fermant la boite aux lettres

c. Vous n'avez pas reçu ma lettre + vous auriez dû la recevoir

d. C'est que j'étais à l'étranger le mois passé

(manière)

(cause)

Dans ces cas d'interprétation autre que de manière/moyen, comment admet une interprétation en tant qu'opérateur qui lie une variable de proposition. De plus, les questions admettent une réponse informative, donc ce ne sont pas nécessairement ou exclusivement des questions rhétoriques au sens de ce terme discuté par Sadock (1971 et 1974), Borillo (1981), Kerbrat-Orecchioni (1991), Han (2002). Pour expliquer ce que c'est qu'une question rhétorique, il a été proposé, par Sadock (1971) et d'autres, d'exploiter la distinction entre l'énonciation d'une phrase, à savoir l'acte locutoire Austinien, et la fonction qu'elle a dans un contexte, à savoir la force illocutoire. En l'occurrence, il y aurait question rhétorique du fait d'un découplage entre forme (interrogative) et fonction (assertive). De plus, les questions rhétoriques positives seraient des questions qui sont associée à la force illocutoire d'une assertion négative et les questions rhétoriques négatives, telles que (2a), auraient la force illocutoire d'une assertion positive. Enfin, la 'réponse' d'une question rhétorique serait connue (selon Rohde, 2009), ce qui les rend triviales. En réalité, notre étude des questions en comment va permettre de voir que la situation est bien plus finement articulée. ${ }^{\mathrm{i}}$

Ensuite, lorsque la question n'interroge pas sur la manière, la phrase est associée avec plusieurs propositions qui ont des statuts différents. Il se peut que comment porte sur la cause d'un processus/événement décrit par une clause souvent enchâssée (sans considérer l'éventuelle modification de manière), dorénavant la proposition préjacente, voir la partie en italique en (3).

\section{(3) Comment ne pas voir qu'elle annonçait d'autres bouleversements?}

Les connaissances des participants à la conversations sont aussi exprimables en termes de propositions, qui sont présupposées ou inférables, selon les cas. On voit donc qu'il faut distinguer la disponibilité ou non d'une lecture de cause, le fait qu'il y ait question ou 
assertion (ou plutôt qu'il y ait des degrés d'engagement différents relativement à la préjacente) et que l'assertion soit positive ou négative.

L'étude de l'articulation de ces lectures et son explicitation est le but de cette contribution. La section 2 nous permet de rappeler quelques éléments du bagage théorique que nous utilisons et quelques hypothèses de base en sémantique formelle que nous reprenons. Ensuite, notre ligne d'attaque est d'examiner quelques possibilités interprétatives qui dérivent de la nature de comment en tant que mot qui interroge à propos d'un ajout souvent rattaché à un prédicat enchâssé, et donc ne questionnant pas forcément la valeur de vérité de la proposition exprimée par la phrase qui le contient, ainsi que d'examiner de plus près le statut de la préjacente et d'éventuelles propositions qui l'englobent au sens large. Premièrement, en section 3, nous introduisons la question de la distribution des lectures et nous considérons les contraintes qui dérivent de la nature des prédicats ou verbes auxiliaires qui enchâssent la préjacente, p.ex. la préjacente décrit l'événement de la venue de Marie en (4).

(4) a. Comment Marie peut-elle venir à la réunion?

b. Comment Marie a-t-elle pu venir à la réunion?

Nous verrons que plusieurs prédicats permettent les lectures de comment non de manière, avec des restrictions. Nous allons parler d'effet d'îlot pour faire référence aux contraintes sur la possibilité d'avoir au moins une lecture de manière et plus en général sur ce qui est questionné par comment, notamment sur son type sémantique. Deuxièmement, en section 4, nous abordons la question du statut de la préjacente, élément souvent fondamental de la définition de question rhétorique. Nous considérons le contexte en tant que connaissances/croyances des agents épistémiques présents dans la situation et nous examinons la question de la valeur de vérité/polarité de la préjacente dans les questions avec interprétation de manière et de cause. La piste que nous explorons est que la question de la polarité de la préjacente doit être abordée d'une manière plus articulée en utilisant différentes formes/degrés de prise en charge du locuteur d'une telle proposition ou de sa négation, et de prise en charge demandée à l'interlocuteur, ce qui nous permet de ne pas exclure un fond de fonction questionnante pour les interprétations rhétoriques et non. Notre approche est construite sur une vision a priori du locuteur relativement à la préjacente, sans faire appel à des présuppositions projetées par une lecture de la question en comment.

\section{Le bagage théorique et les hypothèses de base}

Partons des exemples donnés en (4). Nous faisons l'hypothèse que les questions de ce type sont des questions matrice, qui portent sur un prédicat (explicite ou implicite) qui prend une proposition pour complément. En d'autres termes, comment porte sur la cause d'un processus/événement décrit par la proposition préjacente, ou sur une proposition qui permet d'inclure parmi les caractérisations possibles du monde actuel des mondes où la préjacente est vraie. Il est utile de s'appuyer sur les types sémantiques pour séparer les différentes lectures et sur une approche à la modalité qui utilise les mondes possibles pour traiter de la lecture de cause. On suppose une base modale (Kratzer, 1981, 1991) qui pour (4) se résume à la situation suivante. Le métro est en grève. Marie ne peut donc pas se déplacer par ce moyen de transport. On suppose qu'il lui reste la voiture et le taxi. Cette base modale nous donne toute l'étendue des possibilités offertes à Marie pour venir à la 
réunion. Dans un monde $w$ accessible à $w_{0}$ (le monde réel), elle vient à la réunion en voiture. Dans un autre monde accessible, elle vient en taxi. Dans un autre monde accessible encore elle reste chez elle. Les questions (4a) et (4b), dans leurs lectures "causales", semblent interroger sur le contenu de la base modale. C'est comme si le locuteur se demandait ce qui dans l'arrière-plan rendait possible le fait que Marie vienne à la réunion. Les réponses (5a) et $(5 \mathrm{~b})$ apparaissent alors comme des ajouts (compatibles) à la base modale ou tout du moins comme une mise en lumière d'une proposition de la base modale qui explique la possibilité que Marie vienne à la réunion.

(5) a. Parce qu'elle prend (a pris) un taxi.

b. Parce qu'elle peut (a pu) prendre un taxi.

Dans notre exemple, la réponse «En taxi.» est une réponse sur la manière et correspond à la phrase Marie peut venir en taxi qu'on peut formaliser de la façon suivante, en omettant par souci de simplicité les arguments événementiels, $\exists \mathrm{w} \in \operatorname{Acc}\left(\mathrm{w}_{0}\right)(\operatorname{venir}(\mathrm{m}, \mathrm{w}) \wedge \operatorname{taxi}(\mathrm{m}, \mathrm{w}))$, où taxi $(\mathrm{x}, \mathrm{w})$ signifie : $\mathrm{x}$ prend un taxi dans le monde $\mathrm{w}$ et où Acc est la fonction d'accessibilité associée à la modalité exprimée par le verbe pouvoir. En faisant cette réponse, l'interlocuteur dit notamment qu'il existe un moyen de transport tel que Marie puisse venir par ce moyen de transport, ce qui peut être formalisé en tant que $\exists \mathrm{P} \exists \mathrm{w} \in \operatorname{Acc}\left(\mathrm{w}_{0}\right)(\operatorname{venir}(\mathrm{m}, \mathrm{w}) \wedge \mathrm{P}(\mathrm{m}))$. Une réponse telle que (5a) correspond à la situation suivante : le fait que Marie vienne est rendu possible du fait qu'elle prend un taxi. C'est une réponse à la question "Comment se fait-il que Marie puisse venir ? " La question revient à se demander s'il existe un élément de l'arrière-plan de la conversation qui explique la possibilité pour Marie de venir. C'est une façon de déclarer que le fait que Marie ait pris un taxi fait partie de l'arrière-plan de la conversation. L'existence d'un élément de l'arrière-plan peut être codée de la façon suivante $\exists \mathrm{P} \exists \mathrm{w} \in(\operatorname{Acc} \oplus \mathrm{P}(\mathrm{m}))\left(\mathrm{w}_{0}\right)(\operatorname{venir}(\mathrm{m}, \mathrm{w}))$, où $\mathrm{Acc} \oplus \mathrm{P}(\mathrm{m})$ est la relation d'accessibilité correspondant à l'arrière-plan d'origine augmenté de la proposition $\mathrm{P}(\mathrm{m})$.

Nous avançons l'hypothèse que le locuteur s'interroge sur la condition qui rend possible l'action de Marie. Dans sa connaissance du monde, rien (de saillant) n'indique que Marie puisse venir à la réunion. Autrement dit, pour le locuteur, l'arrière-plan de la conversation n'offre aucune condition qui indique cette possibilité ou qui permet de considérer de façon raisonnable cette possibilité (sauf a considérer que tout est toujours possible, ce qui n'est pas très raisonnable). Mais il n'exclut pas qu'une telle condition puisse exister. Selon l'hypothèse faite ici, la question du locuteur porterait justement sur cette condition (c'est une hypothèse sans doute trop forte mais elle mérite d'être explorée). L'interprétation causale peut donc être ramenée à l'intérieur d'une approche à la sémantique des questions ordinaires tel qu'il a été élaboré par Groenendijk et Stokhof (1997), selon lequel la contribution d'une question est la partition des mondes possibles contextuellement pertinents. Ensuite, le statut de la préjacente et la connaissance dont le locuteur et l'interlocuteur disposent interviennent dans le calcul de la partition à opérer.

\section{Lectures de manière et effets d'îlot}

Dans cette section, nous identifions certains facteurs remarquables, de nature grammaticale ou lexicale, qui rendent difficile, si ce n'est indisponible, la lecture de manière de certaines questions en comment, et nous allons appeler une telle indisponibilité un effet d'îlot de manière. Pour des soucis de simplicité, les questions analysées seront de forme similaire entre elles, à savoir qu'elles contiendront un verbe matrice (ou principal) et une proposition subordonnée dont le verbe sera qualifié d' "enchâssé » (comme dans les exemples 9 et 10). Au préalable, il peut être utile de faire une précision sur ce qu'il faut 
comprendre par lecture de manière. Par ce terme nous entendons l'attribution d'une propriété à une action, attribution qui généralement se fait par le truchement d'un modificateur du VP. Ce n'est donc pas une simple question d'adverbiaux d'une certaine nature, mais plutôt une question de fonction dans la phrase, comme l'a remarqué Van de Velde (2009). Les adverbiaux qui prédiquent une propriété d'un individu et sont orientés vers le locuteur tel que imprudemment en (6b) comprise comme une réponse à (6a), ont un type sémantique différent et ne nous concernent pas, du fait qu'ils ne répondent pas à une interrogation par comment. Il en va de même des adverbiaux orientés sujet, ce qui est illustré par gentiment en (7b) comprise en tant que réponse à (7a). Pour les adverbes de manière orientés résultat, leur orientation bloque toute lecture de manière en dehors du domaine local, voir (8).

(6) a. Comment s'est-il endormi ?

b. \#Il s'est très imprudemment endormi.

(7) a. Comment a-t-il répondu à ma question ?

b. \#Il a gentiment répondu à ma question, car rien ne l'obligeait à le faire (donc ça a été gentil de sa part, bien qu'il ait répondu froidement).

(8) a. Comment a-t-il (\# dit qu'il a) organisé la liste ?

b. Il l'a organisée alphabétiquement.

Venons-en maintenant aux verbes matrice qui nous intéressent pour cerner les îlots de manière. Des verbes comme contester, savoir, ordonner, vouloir, suivis d'une proposition subordonnée, se comportent différemment vis à vis de l'interrogation de manière. La première différence concerne le statut de la proposition subordonnée. Les verbes d'attitude contester et savoir mettent en relation un individu et une proposition. Ces verbes dénotent une attitude, c'est-à-dire un état mental, d'un individu vis-à-vis d'une proposition. Dans la phrase «Jean conteste que Marie est arrivée », l'individu Jean a une attitude négative visà-vis de la vérité de la proposition selon laquelle Marie est arrivée. Cette relation semble créer un effet d'îlot sur la proposition subordonnée et bloquer la possibilité d'interroger la manière du verbe contenu dans cette proposition subordonnée (8d). La question de manière ne peut alors porter que sur le verbe matrice. C'est le cas dans l'exemple (8) où comment ne peut porter que sur la manière de contester ( $9 b$ et $9 c)$ :

(9) a. Comment Jean conteste-t-il que la planète se réchauffe ?

b. Énergiquement ("vraie" manière)

c. Avec des arguments fallacieux (moyen)

d. \# Il conteste que la planète se réchauffe rapidement

e. Parce qu'il ne croit pas le GIEC (cause)

Le verbe savoir bloque également l'interrogation de la manière du verbe enchâssé (10b et $10 \mathrm{c})$. De plus, la question en comment ne peut pas interroger la manière de savoir. Seule la lecture de cause est alors disponible (10d) :

(10) a. Comment Jean sait-il que Marie est arrivée ?

b. \#Il sait que c'est vite

c. \# En voiture (moyen)

d. Parce qu'il l'a aperçue dans la cuisine

Le verbe savoir a la particularité de ne pas «laisser passer » la négation dans le sens suivant. Dans la phrase Jean sait que Marie n'est pas arrivée, on note $p$ la proposition correspondant à Marie est arrivée et $\sim$ p la proposition Marie n'est pas arrivée, i.e. la négation de $p$. On note $R$ la relation que l'individu Jean (noté $j$ ) entretient avec les propositions qu'il sait être le cas. $R(j, \sim p)$ correspond alors à la proposition Jean sait que 
Marie n'est pas arrivée. Dire que le verbe savoir ne laisse pas passer la négation, c'est dire que la proposition $R(j, \sim p)$ n'implique pas $\sim R(j, p)$.

En revanche, le verbe contester semble laisser passer la négation: contester que la planète ne se réchauffe pas semble impliquer de ne pas contester que la planète se réchauffe. La contrainte sur la négation ne peut alors pas servir de test dans l'impossibilité d'interroger la manière du verbe enchâssé. Mais le verbe contester possède une autre propriété, celle de ne pas être monotone croissant dans le sens suivant. Si $p$ désigne la proposition la planète se réchauffe et $q$ la proposition la planète se réchauffe rapidement, dire que Jean conteste que $q$ est le cas, c'est dire qu'il pense que le monde réel $w_{0}$ ne fait pas partie des mondes $q$ (les mondes où $q$ est vraie). Mais il ne pense pas nécessairement que $w_{0}$ ne fait pas partie des mondes $p$. Autrement dit, il ne conteste pas nécessairement que la planète se réchauffe. Le blocage de la négation dans le cas de savoir et la non monotonie dans le cas de contester peuvent être vues comme des contraintes d'îlot qui pourraient jouer un rôle dans l'impossibilité d'interroger la manière du verbe enchâssé ou tout du moins servir de premier test pour repérer l'effet d'îlot sur la manière, spécifiquement pour les verbes d'attitude.

Ces propriétés sur la négation et sur la monotonie ne sont pas partagées par tous les verbes d'attitude. Par exemple, le verbe penser suivi d'une proposition subordonnée ne bloque pas la négation : penser que $p$ n'est pas le cas implique de ne pas penser que $p$ est le cas. De même, le verbe penser respecte une propriété de monotonie croissante : penser que la terre se réchauffe rapidement implique de penser que la terre se réchauffe. Il ne déclenche pas non plus d'effet d'îlot sur la manière, comme le montre l'exemple (11):

(11) a. Comment Marie pense-t-elle que je suis arrivé ?

b. Précipitamment (« vraie » manière)

c. En voiture (moyen)

Les verbes ordonner et vouloir ne dénotent pas une relation entre un individu et une proposition. Pour ces deux verbes, aucun effet d'îlot sur la manière n'est observé.

La question en comment peut alors interroger le verbe enchâssé. Dans l'exemple (12), la question peut interroger la manière du verbe enchâssé (12c). De plus, la manière d'ordonner elle-même peut être interrogée (12b) :

(12) a. Comment Marie ordonne-t-elle que l'on quitte la salle ?

b. Marie ordonne à voix haute/solennellement que l'on quitte la salle

c. Marie ordonne que l'on quitte la salle rapidement

Avec le verbe vouloir, la question en comment peut également interroger la manière du verbe enchâssé (13b). Mais la manière de vouloir ne peut pas être interrogée (13c) :

(13) a. Comment Marie veut-elle que j'accepte l'offre ?

b. Avec le sourire.

c. \# Elle le veut vraiment.

d. Parce qu'elle pense que c'est bien pour toi.

Cependant, parmi les verbes qui ne sont pas des verbes d'attitude, certains verbes créent un effet d'îlot sur la manière. Le verbe s'étonner est un verbe d'émotion plus que d'attitude propositionnelle. Dans l'exemple (14a), la manière du verbe enchâssé n'est pas accessible (14b). Seule la manière du verbe matrice peut alors être interrogée (14c) :

(14) a. Comment Marie s'étonne-t-elle que je sois arrivé ?

b. \# En train / \# à l'heure 
c. En écarquillant les yeux

Quels que soient les critères utilisés pour déterminer les contraintes d'îlot, nous voulons distinguer quatre cas de figure, présentés dans le tableau suivant, en fonction des deux critères suivants :

Critère $1\left(\mathrm{C}_{1}\right)$ : peut-on interroger la manière du verbe matrice ?

Critère $2\left(\mathrm{C}_{2}\right)$ : le verbe matrice déclenche-t-il un effet d'îlot sur la subordonnée?

Tableau 1. Disponibilité de la lecture de manière des question en comment.

\begin{tabular}{c|c|l}
$\mathrm{C}_{1}$ & $\mathrm{C}_{2}$ & Disponibilité de la lecture de manière de la question en comment \\
\hline $\mathrm{O}$ & $\mathrm{O}$ & manière sur le verbe matrice disponible (b) \\
$\mathrm{O}$ & $\mathrm{N}$ & manières sur le verbe matrice et sur le verbe enchâssé disponibles (a) \\
$\mathrm{N}$ & $\mathrm{O}$ & lecture de manière non disponible (6) \\
$\mathrm{N}$ & $\mathrm{N}$ & manière sur le verbe enchâssé disponible (5)
\end{tabular}

Théoriquement, dans les trois cas de figure $(\mathrm{O}-\mathrm{O}),(\mathrm{O}-\mathrm{N})$ et $(\mathrm{N}-\mathrm{N})$, la lecture de manière est disponible, même si le verbe sur lequel on veut interroger la manière (matrice ou enchâssé) est (déjà) modifié par un adverbe ou par un complément de manière. La manière n'est théoriquement jamais saturée. Il est théoriquement possible d'ajouter autant de modificateurs de manière que l'on veut sur un verbe. Dans l'exemple (15), le verbe enchâssé quitter est (déjà) modifié par l'adverbe rapidement. La réponse (15b) semble alors moins naturelle que la réponse (15c) sur le moyen :

(15) a. Comment Marie ordonne-t-elle que l'on quitte la salle rapidement ?

b. ?? Silencieusement

c. Par la fenêtre (moyen)

d. Parce qu'on a largement dépassé l'horaire prévu

La disponibilité réelle de la lecture de manière sur le verbe matrice ou sur le verbe enchâssé dépend alors de critères pragmatiques tels que la présence ou l'absence d'informations (présuppositions) dans le contexte qui légitiment l'interrogation de la manière du verbe matrice ou enchâssé. De plus, la prosodie utilisée par le locuteur peut jouer un rôle et orienter l'interprétation vers une lecture de cause. Ces éléments de contexte et de prosodie seront mis à profit dans la section 4. Inversement, certains verbes tels que se porter sous-catégorisent la manière et forcent la lecture de manière.

Dans cette section, nous avons cerné la (non-)disponibilité de la lecture de manière des questions en comment de plusieurs prédicats à l'aide de tests sur la portabilité de la négation et sur les implications entre formules. La discussion détaillée de ces cas a mis en évidence des chevauchements entre les îlots de manière et un certain nombre d'autres phénomènes en rapport avec la négation et la présupposition et qui concernent les mêmes verbes, mais pas toujours tous. L'un de ces phénomènes est la montée de la négation (de Cornulier 1973, Horn 1978, Tovena 2000), et penser en est une illustration typique. Cependant, contester et ordonner, qui tous les deux ne sont en général pas cités parmi les verbes à montée de la négation, ont un comportement discordant vis-à-vis des îlots de modalité, donc le chevauchement n'est que partiel. Un autre phénomène est celui de la présupposition et de sa distinction d'une implication logique, souvent opérée au moyen de tests tels que la négation, voir Gazdar (1979) et suivants. Un autre encore est celui de la factivité des compléments phrastiques de certains verbes, et un verbe d'attitude tel que savoir et l'émotif-factif s'étonner en sont des illustrations typiques, voir Kiparsky et Kiparsky (1971), Karttunen (1971) et suivants, factivité qui est souvent traitée en tant que 
présupposition de complément phrastique. Or, si la factivité des verbes tels que savoir et $s$ 'étonner peut bien être un ingrédient qui contribue à leur capacité de créer des îlots de manière, cette explication ne s'applique pas à contester, qui pourtant entraîne également un îlot sans factivité. Tout n'est donc pas une question de factivité. Ces points sont sans doute à reprendre dans la suite de nos recherches sur les questions en comment.

Plusieurs lectures de manière ou de cause sont possibles et nous souhaitons consacrer la dernière partie de cet article à l'identification des éléments issus du contenu propositionnel et du contexte capables d'orienter l'interprétation.

\section{Questions en comment et polarité}

Dans cette section, nous souhaitons mettre en lumière des éléments caractéristiques des questions en comment de manière ou de cause, issus du contenu propositionnel de la question, à savoir une préjacente, un contenu modal (à savoir une proposition contenant un modal et la préjacente) et une relation de "polarité » entre ces deux contenus propositionnels. Avant d'expliciter cette relation (sous-section 4.3), nous détaillons les différentes interprétations de manière (sous-section 4.1) et de cause (sous-section 4.2) sur lesquelles nous fondons notre raisonnement. Pour des soucis de clarté, les phrases analysées seront toutes de la forme : comment + verbe modal pouvoir + proposition à l'infinitif.

\subsection{Lectures de manière}

Commençons par la lecture de manière « ordinaire ». Dans l'exemple (16), le contexte est le suivant : le verbe pouvoir est compris relativement aux règles de bonne conduite que Jean doit respecter à l'égard d'un inconnu (modalité déontique) et la question en comment porte sur la manière de parler à un inconnu :

(16) a. A. Comment Jean peut-il parler à un inconnu ?

b. B. Respectueusement

La valeur de vérité de la préjacente (Jean parle à un inconnu) n'affecte pas l'interprétation de la question : l'événement de Jean parlant à un inconnu reste virtuel. En terme de mondes possibles, il existe un monde $w$ accessible à partir du monde réel $w_{0}$ (i.e. compatible avec les règles en vigueur) dans lequel Jean parle à un inconnu d'une certaine manière. Ici, la valeur de la préjacente n'intervient pas dans l'alternance des interprétations de la question en comment (ordinaire, rhétorique, etc.) Notamment, la phrase au passé composé (comment jean a-t-il pu parler à un inconnu) actualise l'événement de Jean parlant à un inconnu sans pour autant orienter la lecture vers un type d'interprétation. De plus, dans cette lecture, on suppose que le locuteur $\mathbf{A}$ ne fait aucune présupposition négative sur la possibilité de Jean de parler à un inconnu (plus exactement, il ne fait pas de présupposition de polarité opposée, à savoir Jean ne peut pas parler à un inconnu pour (16a), ou Jean peut parler à un inconnu si (16a) était Comment Jean peut-il ne pas parler à un inconnu?). On suppose qu'il ne fait pas non plus de présupposition négative sur l'existence d'une manière (pour Jean) de parler à un inconnu. La question est alors une simple recherche d'information sur la manière dont Jean peut parler à un inconnu.

«Complétion » du jugement: Le locuteur porte un jugement a priori sur l'existence d'une manière qui satisfait la préjacente. Il peut être « agnostique » sur cette existence : ou bien il n'exclut pas l'existence d'une manière (qu'il ne connaît pas), ou bien il pense qu'une manière (qu'il ne connaît pas) existe. Ce jugement sur l'existence induit un jugement sur la possibilité que la préjacente soit satisfaite : dans un premier cas, il n'exclut pas cette possibilité et c'est la réponse à la question qui lui permettra de trancher a posteriori ; dans 
un deuxième cas, il juge que la préjacente est possible et la réponse lui permettra de fonder son jugement a posteriori. Dans les deux cas, il s'agit d'un possible a priori et le locuteur cherche une information (une manière) qui conforte ce jugement de possibilité. Une réponse congruente à la question permet au locuteur de compléter son jugement de possibilité.

Cependant, dans certains contextes, le locuteur fait une présupposition négative sur l'existence d'un moyen et la question devient rhétorique. Le contexte de l'exemple (17) est le suivant : au cours d'une conversation avec Marie, Jean a proféré des accusations non fondées à son égard. Le locuteur A estime a priori qu'il n'existe aucun moyen pour Jean de se faire pardonner. Il pose une question sur le moyen de se faire pardonner sous la forme d'un défi à son interlocuteur de trouver un moyen valable. Le jugement du locuteur A n'est pas révisable. L'interlocuteur $\mathbf{B}$ tente une réponse que le locuteur $\mathbf{A}$ refuse (17c) :

(17) a. A. Comment Jean peut-il rattraper son erreur maintenant ?

b. B. En présentant ses excuses? (moyen)

c. A. Ce n'est pas suffisant.

d. A. Oui, tu as raison.

Dans cet exemple, le locuteur présuppose qu'il n'existe aucune manière pour Jean de se faire pardonner. C'est une façon de communiquer l'impossibilité pour Jean de se faire pardonner.

«Révision » du jugement: ici, le locuteur juge qu'il n'existe pas de manière qui satisfasse la préjacente et il juge par ailleurs que la connaissance sur laquelle il fonde ce jugement est complète. Il s'agit alors d'un jugement d'un impossible non révisable. En posant la question, il veut mettre son interlocuteur dans l'impossibilité de trouver la manière qui satisfasse la préjacente. C'est une façon pour le locuteur de communiquer l'impossibilité de la préjacente voire la négation de la préjacente.

Passons maintenant à la lecture de manière « ordinaire» avec présupposition. Si le locuteur A estime a priori qu'il n'existe aucune manière pour Jean de se faire pardonner (parce qu'aucune manière ne lui vient à l'esprit) mais qu'il reste ouvert à cette possibilité, alors il cesse de mettre au défi son interlocuteur de trouver une manière. Il recherche une information qui lui permettra de réviser son jugement. La tentative de réponse de son interlocuteur (17b) pourra être acceptable à ses yeux (17d). La question cesse d'être rhétorique. C'est une question non rhétorique sur la manière avec une présupposition d'impossibilité révisable, malgré une quelque nuance de rhétorique.

«Révision » du jugement : ici, le locuteur juge a priori, en fonction de l'étendue de sa connaissance, qu'il n'existe pas de manière qui satisfasse la préjacente mais il n'exclut pas pour autant que son interlocuteur ait connaissance d'une telle manière. Son jugement est alors celui d'un impossible révisable. Autrement dit, il sait qu'il fonde son jugement relativement à une connaissance partielle et il cherche l'information qui lui permettra d'étendre sa connaissance et de réviser son jugement.

\subsection{Lectures de cause}

Pour l'exemple (18), repris de l'exemple (16), les lectures de manière et de cause sont disponibles (cf. section 3 ) mais le contexte est moins favorable à une lecture de manière : A pense dur comme fer que Jean est très timide et qu'il n'oserait jamais parler à un inconnu. B semble pourtant constater que Jean est en train de parler à un inconnu (18a). A met en question la réalité ou la possibilité de cet événement (18b) :

(18) a. B. Regarde ! Jean parle à un inconnu ! 
b. A. Comment Jean peut-il parler à un inconnu ?

c. B. Parce qu'il n'est pas si timide que ça!

d. A. Ce n'est pas un inconnu, c'est son ami Paul.

La question (18b) peut être interprétée de plusieurs façons. Dans une première interprétation de cause, qu'on pourrait appeler ordinaire, le locuteur $\mathbf{A}$ accepte la réalité de l'événement (Jean parle à un inconnu) et se demande quelle condition rend possible cet événement. Pour le locuteur $\mathbf{A}$, Jean ne peut a priori pas parler à un inconnu. Il recherche donc une information qui lui permette de revoir ou réviser son jugement. Son interlocuteur B lui donne alors cette information (18c).

Dans une deuxième interprétation de cause, qui a plus un goût rhétorique, le locuteur $\mathbf{A}$ réfute la réalité de l'événement et met au défi son interlocuteur de prouver la possibilité de cet événement. Il refuse également l'explication donnée par $\mathbf{B}$ en (18c) et prouve que l'événement de Jean parlant à un inconnu n'a pas lieu : Jean parle à une personne connue (18d). La question posée par A en (18b) ne constitue pas une recherche d'information. C'est question rhétorique qui permet à $\mathbf{A}$ d'exprimer son refus de l'hypothèse que l'événement est bien réel. Dans la première interprétation, le locuteur $\mathbf{A}$ est prêt à revoir son jugement et il recherche l'information qui lui permettra de le faire. Dans la deuxième interprétation, il n'est pas prêt à revoir son jugement et il met au défi son interlocuteur de trouver une raison de lui faire changer son jugement. Dans ce dernier cas, une façon de désambiguïser l'interprétation est d'utiliser le conditionnel : «comment Jean pourrait-il parler à un inconnu? »

Il y a une troisième interprétation de (18b) qui représente une deuxième lecture de cause « rhétorique ». On reprend la question comment Jean peut-il parler à un inconnu ? (18b) mais dans un contexte déontique : le locuteur juge qu'il n'est pas acceptable que Jean parle à un inconnu. Dans le même temps, il constate que Jean parle à un inconnu. Il ne cherche pas d'information pouvant modifier son jugement déontique. C'est une question rhétorique qui lui permet de communiquer son jugement déontique a priori, à savoir qu'il est inacceptable (pour Jean) de parler à un inconnu.

\subsection{Contenu propositionnel et polarité}

Dans cette sous-section, nous partons des questions en comment exposées aux soussections 4.1 et 4.2 et nous mettons en lumière deux éléments de leur contenu propositionnel et la relation que ces deux éléments entretiennent. Ces questions contiennent le modal pouvoir suivi d'une proposition infinitive. Le premier élément que nous souhaitons extraire du contenu propositionnel est la préjacente. Rappelons qu'une question en comment de cause interroge la cause d'un événement et que la préjacente est la proposition qui décrit cet événement. Dans la question (18b) la préjacente est la proposition correspondant à l'événement Jean parle à un inconnu.

Le deuxième élément que nous souhaitons extraire du contenu propositionnel de la question en comment est la proposition modale. La proposition modale est la composition de la modalité appliquée à la préjacente. Dans la question (18b), la modalité explicite (pouvoir) concerne la capacité de Jean. La proposition modale correspond à Jean peut parler à un inconnu. Mais la modalité peut être implicite comme dans la question (19) qui interroge également la cause de l'événement Jean parle à un inconnu, i.e. les conditions qui rendent possible cet événement. Comme dans l'exemple (18), cette possibilité concerne la capacité de Jean et la modalité implicite est précisément cette possibilité :

(19) Comment Jean a-t-il parlé à un inconnu ? (cause avec modal implicite) 
Nous définissons également la préjacente et la proposition modale dans le cas d'une question en comment de manière construire avec un modal et une proposition enchâssée correspondant à l'événement dont la question interroge la manière (20a). La préjacente est alors la proposition enchâssée (Jean parle à un inconnu) et la proposition modale (Jean peut parler à un inconnu) est la composition de la modalité (déontique) appliquée à la préjacente (20).

(20) a. A. Comment Jean peut-il parler à un inconnu ? (manière)

b. B. Respectueusement

La proposition modale peut être exprimée en termes de situations ou de mondes possibles. Pour (20a) : il existe un monde possible (compatible avec les règles en vigueur) dans lequel Jean parle à un inconnu, i.e. dans lequel la préjacente (Jean parle à un inconnu) est satisfaite.

Examinons maintenant le jugement du locuteur vis-à-vis de la préjacente et de la proposition modale et la relation entre ces deux jugements qui en découle. Nous prenons en considération les lectures de cause en premier. Dans une lecture ordinaire de cause de la question (18b), le locuteur n'a pas toutes les informations lui permettant de juger que Jean a la capacité de parler à un inconnu. Autrement dit, il ne juge pas a priori vraie cette proposition modale. Dans le même temps, il peut accepter la vérité de la proposition préjacente Jean parle à un inconnu, soit parce qu'il le constate, soit parce qu'on lui rapporte, soit parce qu'il le conçoit. Ces deux jugements (proposition modale non a priori vraie et préjacente potentiellement vraie) sont dans une relation d'antagonisme. Cet antagonisme n'est pas une simple opposition vrai/faux. Le premier jugement tend vers la réfutation de la proposition modale alors que le deuxième jugement tend vers l'acceptation de la préjacente. Nous parlerons d'une polarité de jugements dont les deux pôles sont constitués par les deux jugements antagonistes. La réponse parce qu'il n'est pas si timide que ça $(18 \mathrm{c})$ est alors une façon de faire disparaître cet antagonisme ou cette polarité. La nouvelle information apportée par l'interlocuteur permet au locuteur de revoir son jugement initial et d'accepter la vérité de la proposition modale (Jean peut parler à un inconnu).

Passons maintenant à la version rhétorique de la question de cause, dont deux cas sont à identifier. Dans une première lecture de cause rhétorique, le locuteur n'accepte pas le jugement selon lequel la préjacente (Jean parle à un inconnu) est vraie. Le fait que Jean parle à un inconnu est une hypothèse que le locuteur prête à autrui (son interlocuteur par exemple) mais qu'il souhaite écarter. De plus, il juge que Jean n'a pas la capacité de parler à un inconnu (proposition modale fausse). Ces deux jugements, qui traduisent les attentes (expectations) du locuteur, créent un antagonisme entre une préjacente hypothétiquement vraie (jugée comme telle par autrui) et une proposition modale non satisfaite. Avec sa question, le locuteur attend une confirmation de la fausseté de la préjacente. Dans la réponse ce n'est pas un inconnu, c'est son ami Paul (18d), il donne lui-même cette confirmation. Dans une deuxième lecture de cause rhétorique, dans un contexte déontique, le locuteur juge que la préjacente Jean parle à un inconnu est vraie dans le monde réel mais il juge que c'est inacceptable. Ses deux jugements sont clairement antagonistes. C'est peutêtre de cet antagonisme qu'il est question dans les traitements des questions rhétoriques qui invoquent un acte illocutoire assertif de polarité opposée à la forme de la préjacente, voir Sadock et les références indiquées à la section 1 .

La situation est différente pour ce qui est des lectures de manière. Une question en comment de manière ordinaire ne donne lieu à aucun antagonisme. Dans la question (20a), le locuteur juge déontiquement acceptable que Jean parle à un inconnu et il se demande de quelle manière Jean parlerait à un inconnu dans une situation où Jean respecterait les règles en vigueur. Dans cet exemple, la valeur de vérité de la préjacente dans le monde réel n'entre pas en considération. Si on met le modal au passé composé (comment Jean a-t-il pu 
parler à un inconnu?), la valeur de la préjacente est actualisée (vraie dans le monde réel), sans altérer fondamentalement le sens de la question. Autrement dit, aucun antagonisme entre les deux jugements (sur la proposition modale et sur la préjacente) n'intervient dans la lecture de la question. Il semble également qu'il n'y a aucun antagonisme dans une question en comment de manière rhétorique. Dans la question (21), reprise de l'exemple (17), le locuteur juge a priori qu'il n'existe aucun moyen (pour Jean) de rattraper son erreur. La valeur de la préjacente n'intervient pas sur le type de lecture. Cependant, en (21) il peut y avoir un biais de la part du locuteur vers une réponse de polarité opposée à la forme de la question. Dans le cas typique d'une question formulée de manière positive, la réponse attendue est un ensemble vide de possibilités de se rattraper.

\section{(21) Comment Jean peut-il rattraper son erreur maintenant ?}

Enfin, les questions de manière permettent un autre type de lecture rhétorique, qui n'a pas encore été mentionné jusqu'ici, notamment le cas que Rohde (2009) appelle 'non-null answer' (réponse non-nulle) et qui correspond à la situation où il y a une réponse précise et évidente qui est visée, donc la réponse est un ensemble singleton et sa polarité n'est pas opposée à celle de la forme de la question. Par exemple, dans une situation où Jean est connu de tout le monde pour son caractère de soumission avilissante, la question sous (22) peut être utilisée et viser une réponse de type 'Servilement, comme d'habitude'.

(22) Comment Jean a répondu à la dernière requête excessive de son chef?

$\mathrm{Au}$ final, l'existence d'une polarité de jugements semble une particularité des questions en comment de cause, qu'elles soient ordinaires ou rhétoriques. Nous voulons utiliser cette particularité pour mieux comprendre le mécanisme interprétatif des questions en comment de cause.

Reprenons la question comment Jean peut-il parler à un inconnu? On suppose que la modalité est déontique (est-il acceptable de parler à un inconnu au regard des règles en vigueur?) C'est un élément du contexte crucial pour l'interprétation. Mais en dehors de tout autre élément du contexte et en l'absence de toute prosodie, les lectures de manière et de cause restent disponibles. Supposons maintenant que le contexte ne soit pas favorable à la lecture de manière. Par exemple, la question de manière ne fait pas sens dans le contexte, i.e. elle n'est pas pertinente dans le contexte. Supposons de plus que le locuteur utilise une prosodie qui marque sont étonnement. Ces deux éléments peuvent suffire à orienter l'interprétation de la question vers une question de cause. Or une lecture de cause donne systématiquement lieu à une polarité de jugements. L'interlocuteur reçoit donc l'information qu'il existe (pour le locuteur) un antagonisme entre la proposition préjacente (Jean parle à un inconnu) et la proposition modale (Jean peut parler à un inconnu). À ce stade de l'interprétation, aucune valeur n'est assignée à ces deux jugements (i.e. deux pôles) et l'interprétation n'est pas complète. Pour que l'interprétation réussisse, l'interlocuteur doit pouvoir assigner des valeurs à ces deux pôles à partir d'éléments du contexte. Par exemple, dans un contexte où le fait que Jean parle à un inconnu a été rapporté, la préjacente (le premier pôle) pourra être interprétée comme potentiellement vraie (polarité positive). La valeur de la proposition modale (le deuxième pôle) sera alors interprétée comme une polarité négative. Plusieurs polarités négatives sont envisageables (allant de non a priori vrai mais révisable à faux non révisable). Si rien dans le contexte ne laisse penser que le locuteur refuse de revoir son jugement, la proposition modale sera interprétée comme non a priori vraie. L'interprétation qui en résulte est que le locuteur recherche une information qui lui permette de réviser son jugement et d'accepter la possibilité que Jean parle à un inconnu. 
Mais une prosodie proche de l'exclamation qui montre le refus du locuteur de revoir son jugement orientera le deuxième pôle vers une autre valeur, à savoir une proposition modale fausse non révisable. Comme la modalité est supposée déontique, l'interprétation qui en résulte est alors que le locuteur communique un jugement selon lequel il est inacceptable que Jean parle à un inconnu.

\section{Conclusion}

Dans cette contribution, nous avons discuté de plusieurs cas où comment n'interroge pas sur la manière mais a une lecture parfois appelée de cause. Nous avons mis en clair la différence entre une interrogation sur la valeur possible de la proposition préjacente dans le monde d'évaluation et une caractérisation rhétorique traditionnelle.

Il y a des affinités entre les prédicats qui enchâssent la préjacente dans une lecture de cause et les prédicats à montée de la négation et les prédicats factifs, mais ces caractérisations ne parviennent pas, à elles-mêmes, à exprimer les contraintes qui régissent les îlots de manière. Notre discussion a fait progresser l'étude du phénomène au delà de l'analyse syntaxique proposée par Sprouse (2007). En effet, nous avons mis en avant comment plusieurs facteurs de la nature des prédicats qui enchâssent la préjacente orientent l'interprétation de la question dans une palette d'interprétations qui ne constitue pas pour autant un continuum. Il y a rupture entre les interprétations de manière, de cause et la lecture rhétorique.

De plus, l'appellation 'rhétorique' se trouve enrichie de contenu. Nous avons précisé en quoi certaines lectures des questions en comment peuvent avoir été appelées 'rhétoriques' en abordant la question du statut de la préjacente, élément souvent fondamental de la définition de question rhétorique, à l'intérieur d'une vue du contexte en tant que connaissances/croyances des agents épistémiques présents dans la situation.

L'interprétation des questions en comment de cause, qu'elles soient 'rhétoriques' ou non, se fonde sur l'établissement d'une polarité de jugements, inhérente à ce type de question, entre un jugement sur la préjacente et un jugement sur la proposition modale. Les informations du contexte permettent alors l'assignation des valeurs de cette polarité et in fine l'interprétation de la question en comment.

L'étude présentée se base sur un petit corpus et nous envisageons d'utiliser les tests identifiés (sur la négation, sur la monotonie, etc.) ainsi que le calcul de l'interprétation des questions en comment de cause en fonction de la polarité, dans un corpus plus conséquent.

De plus, une étude lexicale et modale plus approfondie, notamment des verbes d'attitude, devrait permettre de mieux comprendre la distribution des effets d'îlot de manière.

\section{Références}

Borillo, A. (1981). Quelques aspects de la question rhétorique en français. DRLAV Revue de Linguistique, 25, 1-33.

Collins, C. (1991). Why and how come. MIT Working Papers in Linguistics, 15, 31-45.

Cornulier, B. de, (1973). Sur une règle de déplacement de négation. Le Français Moderne, 41, 43-57.

Cornulier, B. de, (1974). Pourquoi et l'inversion du sujet non clitique. In Actes du Colloque Franco-Allemand de Grammaire Transformationnelle, volume I. Etudes de syntaxe, 139-163, Tübingen : Max Niemeyer Verlag.

Desmets, M. et A. Gautier. (2009). 'Comment n’y ai-je pas songé plus tôt?', questions 
rhétoriques en comment. Travaux de linguistique, 58(1), 107-125.

Fleck, F. (2008). Interrogation sur la manière, interrogation sur la cause et négation : existet-il des interrogations négatives portant sur la manière?. De lingua Latina, 1, 1-7.

Gazdar, G. (1979). Pragmatics. New York : Academic Press.

Groenendijk, J. et M. Stokhof. (1997). Questions. In J. van Benthem et A.G.B. ter Meulen, (dirs.), The handbook of logic and language, 1055-1124. Cambridge USA : The MIT Press.

Han, C.-h. (2002). Interpreting interrogatives as rhetorical questions. Lingua, 112(3), 201229.

Horn, L. (1978). Remarks on Neg-raising. In Pragmatics (Syntax and Semantics 9). P. Cole, (dir.), 129-220. New York: Academic Press.

Hsiao, P.-Y. K. (2017). Rhetorical wh-questions in Chinese and feature movement. UST Working Papers in Linguistics, 9, 1-19

Karttunen, L. (1971). Implicative verbs. Language, 47, 340-358.

Kerbrat-Orecchioni, C. (dir.) (1991). La question. Presses universitaires de Lyon, Lyon.

Kiparsky, C. et Kiparsky, P. (1971). Fact. In Steinberg, D. et Jakobovitz, L. (dirs.) Semantics: An Interdisciplinary Reader, 345-369. Cambridge University Press.

Kleiber, G. (2012). Sur la présupposition. Langages, 2, 21-36.

Korzen, H. (1990). Pourquoi pourquoi est-il différent? l'adverbial de cause et la classification des adverbiaux en général. Langue française, 88, 60-79.

Kratzer, A. (1981). The notional category of modality. In Eikmeyer, H. et Rieser, H., (dirs.), Words, Worlds and Contexts, 38-74. Berlin: de Gruyter.

Kratzer, A. (1991). Modality. In von Stechow, A. et Wunderlich, D. (dirs.), Semantik. Ein internationales Handbuch der zeitgenossischen Forschung, 639-650, Berlin: de Gruyter.

Rohde, H. (2006). Rhetorical questions as redundant interrogatives. San Diego Linguistics Papers, 2, 134-168.

Sadock, J. M. (1971). Queclaratives. In Papers from the Seventh Regional Meeting of the Chicago Linguistic Society, 223-232.

Sadock J. M. (1974). Toward a Linguistic Theory of Speech Acts. New York: Academic Press.

Sprouse, J. (2007). Rhetorical questions and wh-movement. Linguistic Inquiry, 38(3), 572580.

Tovena, L. M. (2000). Neg-raising: negation as failure. In Perspectives on Negation and Polarity Items, Hoeksema, J. et Rullman, H. et Sánchez Valencia, V. et van der Wouden, T. (dirs.), 331-356. Amsterdam \& Philadelphia: John Benjamins.

Tsai, W.-T. D. (2008). Left periphery and how-why alternations. Journal of East Asian Linguistics, 17, 83-115.

Van de Velde, D. (2009). Comment, manières d'être et manières de faire. Travaux de linguistique, 58(1), 39-61. 
i L'étude se base sur des phrases reprises aux linguistes cités dans le texte et sur des données naturalistes et en partie extraites de l'intégralité des textes de Frantext postérieurs à 2009, à savoir un total de 3000969 mots (novembre 2017). Cette collection de textes contient 314 occurrences de questions en comment, dont 82 ont une lecture de cause. Nous remercions Théa Mangatal pour son aide dans l'exploitation de Frantext. 